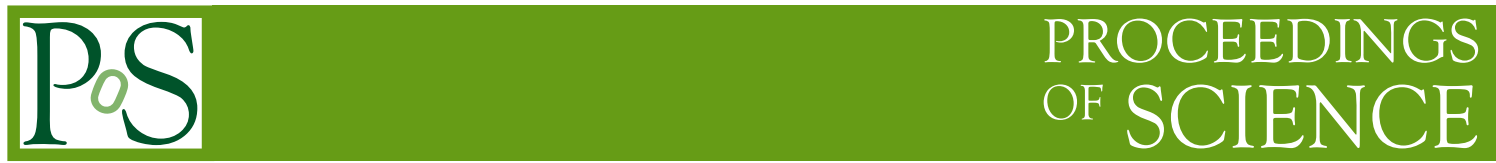

\title{
Hadron resonance gas with repulsive interactions and baryon rich matter
}

\section{P. Huovinen}

Institute of Theoretical Physics, University of Wroclaw, 50204 Wrocław, Poland

E-mail: pasi.huovinen@ift.uni.wroc.pl

\section{P. Petreczky*}

Physics Department, Brookhaven National Laboratory, Upton, NY 11973, USA E-mail:

petreczkabnl.gov

We discuss the role of repulsive baryon interactions in the thermodynamics of hadron gas using the mean-field approach and virial expansion. We calculate the equation of state in the hadron resonance gas framework at non-zero baryon density corresponding to baryon chemical potential $\mu_{B} \leq 400 \mathrm{MeV}$ using the repulsive mean-field approach.

Critical Point and Onset of Deconfinement - CPOD2017

7-11 August, 2017

The Wang Center, Stony Brook University, Stony Brook, NY

\footnotetext{
* Speaker.
} 


\section{Introduction}

QCD thermodynamics at net zero baryon density below the chiral crossover can be described by hadron resonance gas (HRG) model. Equation of State and second order fluctuations of conserved charges obtained in lattice QCD agree well with HRG predictions (see e.g. Refs. [1,2]). The HRG model can be justified using the relativistic virial expansion. In most cases after summation over spin and isospin channels only resonance contributions survive in the second virial coefficient, and thus the interacting gas of hadrons can be approximated as the gas of non-interacting hadrons and hadronic resonances [3]. However, not all hadronic interactions can be treated this way. One example is the nucleon-nucleon interactions, which are non-resonant.

For higher order fluctuations of conserved charges the agreement between the lattice and HRG is no longer good for temperatures close to the chiral transition. It has been argued that this is due to repulsive baryon-baryon interactions $[4,5,6]$. As the baryon density increases the role of repulsive interactions will increase. Therefore in order to extend HRG to non-zero baryon density repulsive interactions have to be included in the description. In this contribution we will do this using the virial expansion and the mean field approach. In the next section we will review the virial expansion for the nucleon gas and the repulsive mean-field. In section 3 we will study the equation of state at non-zero baryon density within the repulsive mean field approach. Finally in section 4 our conclusions will be presented.
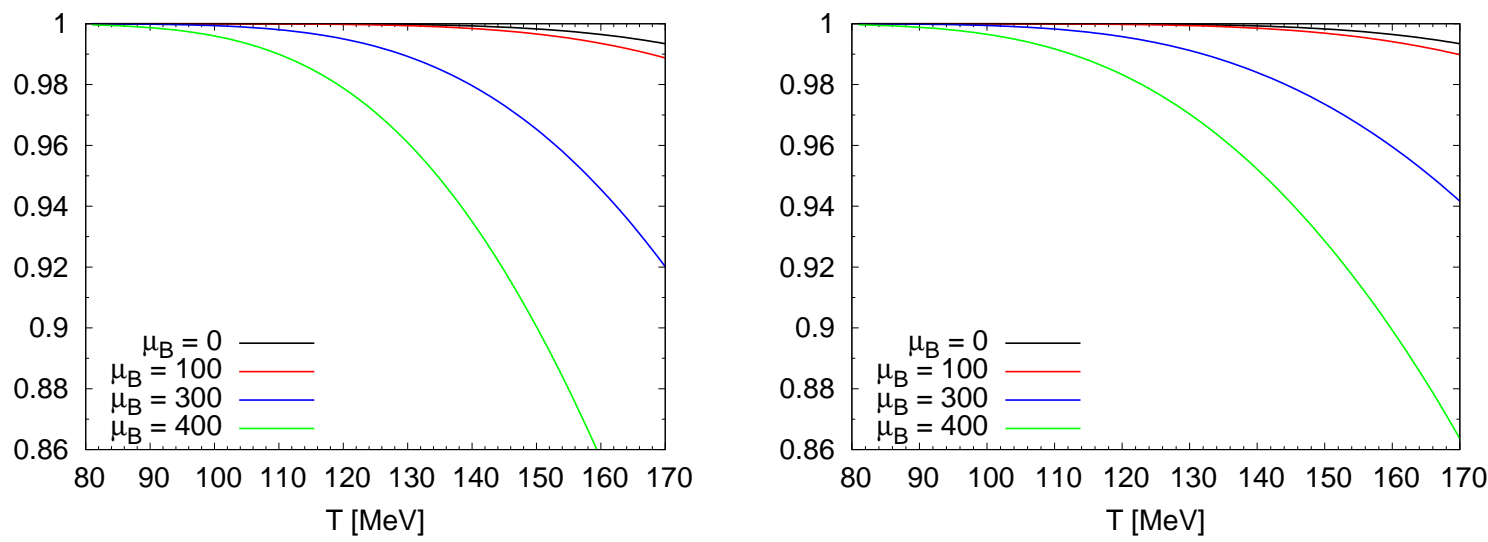

Figure 1: The pressure normalized by the ideal HRG pressure for $\mu_{S}=0$ (left) and $n_{S}=0$ (right) as function of the temperature for different values of $\mu_{B}$ in $\mathrm{MeV}$

\section{Nucleon gas with repulsive mean-field and virial expansion}

Using the virial expansion the pressure of the nucleon gas can be written as [7]

$$
p(T, \mu)=p_{0}(T) \cosh (\beta \mu)+2 b_{2}(T) T \cosh (2 \beta \mu), \beta=1 / T \text {. }
$$

Here

$$
p_{0}(T)=\frac{4 M^{2} T^{2}}{\pi^{2}} K_{2}(\beta M)
$$



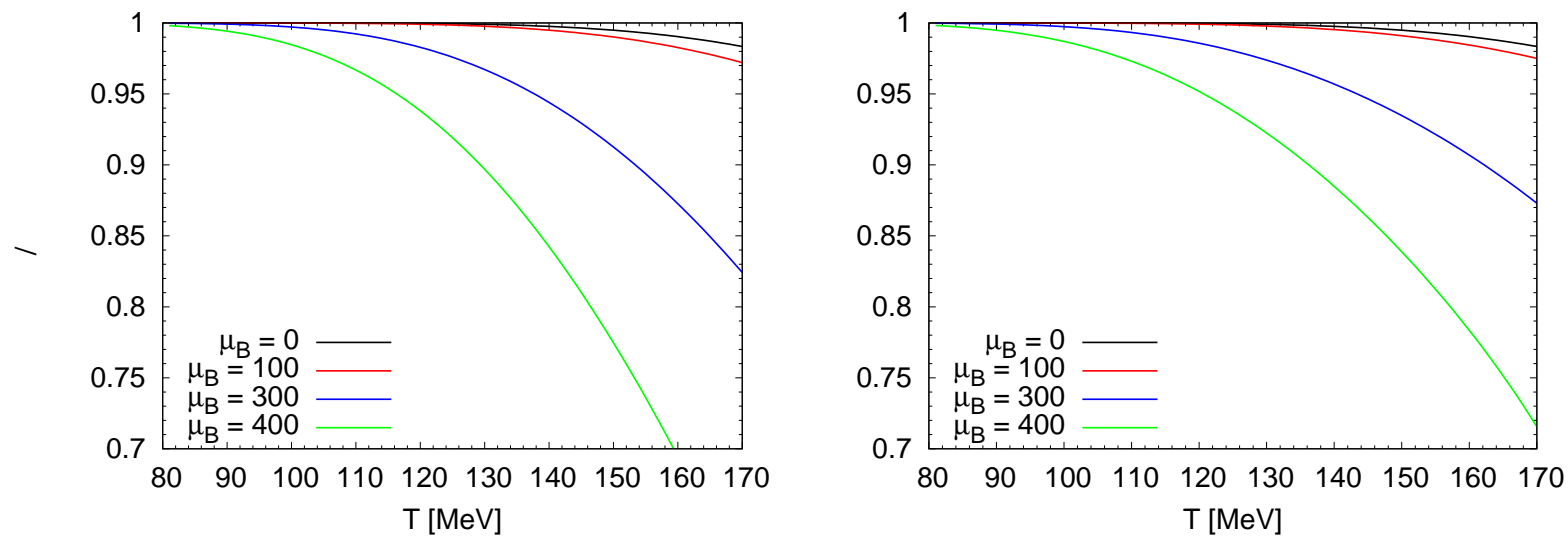

Figure 2: The energy density normalized by the ideal HRG energy density for $\mu_{S}=0$ (left) and $n_{S}=0$ (right) for different values of $\mu_{B}$ in $\mathrm{MeV}$

is the pressure of free nucleon gas at zero chemical potential and the second virial coefficient can be written as

$$
b_{2}(T)=\frac{2 T}{\pi^{3}} \int_{0}^{\infty} d E\left(\frac{M E}{2}+M^{2}\right) K_{2}\left(2 \beta \sqrt{\frac{M E}{2}+M^{2}}\right) \frac{1}{4 i} \operatorname{Tr}\left[S^{\dagger} \frac{d S}{d E}-\frac{d S^{\dagger}}{d E} S\right],
$$

with $S$ being the scattering $S$-matrix and $E$ is the kinetic energy in the lab frame. Furthermore, $M$ is the nucleon mass and $K_{2}(x)$ is the Bessel function of second kind. Using the parameterization of the $S$-matrix in terms of the phase shifts and their numerical values the virial coefficient $b_{2}(T)$ can be calculated, and it turns out that $b_{2}(T)$ is negative [7]. For the comparison with the mean-field approach discussed below it is convenient to write the above expression for the pressure in the following form

$$
p(T, \mu)=p_{0}(T)\left(\cosh (\beta \mu)+\bar{b}_{2}(T) K_{2}(\beta M) \cosh (2 \beta \mu)\right),
$$

with

$$
\bar{b}_{2}(T)=\frac{2 T b_{2}(T)}{p_{0}(T) K_{2}(\beta M)} .
$$

being the reduced virial coefficient.

In the mean field approach the pressure can be written as [7].

$$
p(T, \mu)=T\left(n_{b}+\bar{n}_{b}\right)+\frac{K}{2}\left(n_{b}^{2}+\bar{n}_{b}^{2}\right),
$$

where $n_{b}$ and $\bar{n}_{b}$ are the densities of nucleon and anti-nucleons, respectively, defined by the following self-consistent relations:

$$
n_{b}=4 \int \frac{d^{3} p}{(2 \pi)^{3}} e^{-\beta\left(E_{p}-\mu+U\right)}, \bar{n}_{b}=4 \int \frac{d^{3} p}{(2 \pi)^{3}} e^{-\beta\left(E_{p}+\mu+\bar{U}\right)}, E_{p}^{2}=p^{2}+M^{2} .
$$

Here $U=K n_{b}$ and $\bar{U}=K \bar{n}_{b}$ are the mean-field potentials for nucleons and anti-nucleons. The chemical potential corresponding to the net nucleon density is denoted by $\mu$. The above form 
of the pressure ensures thermodynamic consistency, i.e. $\partial p / \partial \mu=n_{b}-\bar{n}_{b}$. Since we are mostly interested in the region of not too high baryon densities one can expand the above expressions in $\beta U=\beta K n_{b}$ and $\bar{U}=K \bar{n}_{b}$ and keep only the leading order terms in $K$. Then we get

$$
p(T, \mu)=T\left(n_{b}^{0}+\bar{n}_{b}^{0}\right)-\frac{K}{2}\left(\left(n_{b}^{0}\right)^{2}+\left(\bar{n}_{b}^{0}\right)^{2}\right),
$$

with $n_{b}^{0}\left(\bar{n}_{b}^{0}\right)$ being the free nucleon(anti-nucleon) densities. The above equation can also be written as

$$
p(T, \mu)=\frac{4 T^{2} M^{2}}{\pi^{2}} K_{2}(\beta M) \cosh (\beta \mu)-4 K \frac{T^{2} M^{4}}{\pi^{4}} K_{2}^{2}(\beta M) \cosh (2 \beta \mu) .
$$

Now we see that the virial expansion and the expanded mean-field approach give very similar results. From the comparison of these two results one can determine the value of $K$ in a limited temperature range. Since $\bar{b}_{2}(T)$ turns out to be negative, $K>0$, i.e. the mean-field is repulsive. In what follows we will use the value $K=450 \mathrm{MeV} / \mathrm{fm}^{3}$, which is consistent with $\bar{b}_{2}(T)$ at the highest temperatures [7]. It is straightforward to generalize the mean-field approach described above to a multicomponent system, e.g. HRG if one assumes that the repulsive mean-field is the same for all baryons [7]. Within this approach one can estimate the difference between the second order fluctuations and correlations of conserved charges and the fourth and sixth order fluctuations and correlations. The model can explain these differences reasonably well [7] if one assumes that only ground state octet and decuplet baryons are affected by the repulsive interactions.

\section{Equation of state and the freeze-out temperature}

Using extension of the mean-field approach to the multicomponent hadron gas as discussed above it is possible to calculate the equation of state at non-zero baryon chemical potential, $\mu_{B}$ and strangeness chemical potential, $\mu_{S}$. We calculate the pressure using HRG with repulsive mean-field at non-zero baryon and strangeness chemical potentials. We use all the strange and non-strange states from particle data group (PDG) summary tables in HRG calculations. As in Ref. [7] we only include the repulsive mean-field for the ground state octet and decuplet baryons. We will consider temperatures up to $170 \mathrm{MeV}$ and moderate values of the baryon chemical potential $\mu_{B} \leq 400 \mathrm{MeV}$. For these values the meson pressure is larger than the baryon pressure. Therefore, we will use the expanded version of the mean field approach, as discussed above. For $\mu_{B}>400 \mathrm{MeV}$ the matter become baryon dominated. For non-zero $\mu_{B}$ the net strangeness $n_{S}$ is also non-zero. By adjusting the strange chemical potential we get $n_{S}=0$. We calculate the pressure for $\mu_{S}=0$ as well as for $n_{S}=0$.

Our results are shown in Fig. 1 in terms of the ratio of the pressure to the pressure of free HRG, i.e. HRG with no repulsive interactions. For $\mu_{B}=0$ this ratio is smaller than one by a few percent. As we increase the baryon chemical potential this ratio is decreasing, i.e. the effects of repulsive interactions become more important and increase with increasing temperature. For baryon chemical potential of $400 \mathrm{MeV}$ the effect of repulsive interactions are about $14-20 \%$ at the highest temperature. The effect of repulsive interactions is somewhat smaller for the case of net zero strangeness.

In Fig. 2 we show our results for the energy density as the functions of the temperature for $\mu_{S}=0$ and $n_{S}=0$. The energy density is normalized by the free HRG result. As in the case of 

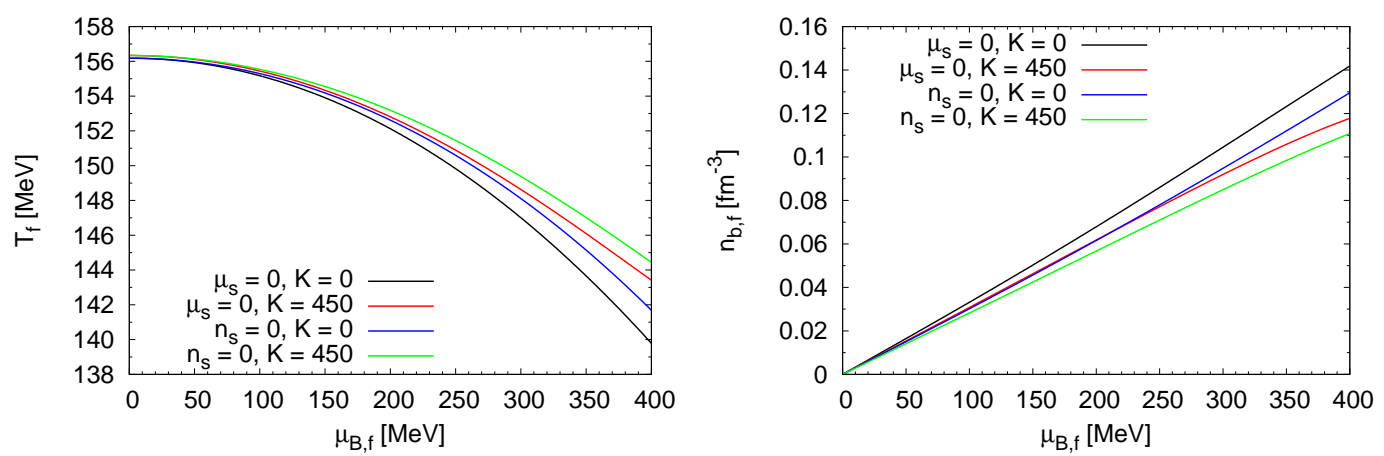

Figure 3: The freeze-out temperature (left) and the net baryon density (right) corresponding to energy density $330 \mathrm{MeV} / \mathrm{fm}^{3}$ as function of $\mu_{B}$. The results are shown for the effect of the repulsive mean-field included or excluded and for $\mu_{S}=0$ and $n_{S}=0$.

the pressure the effect of the repulsive interactions is few percent for $\mu_{B}=0$ and increases with increasing the baryon chemical potential. However, the effect of the repulsive interactions is larger than in the case of the pressure. At the highest temperature the effect of repulsive interactions are as large as $30 \%$. Again the effect of the repulsive interactions is smaller for the $n_{S}=0$ case than for the $\mu_{S}=0$ case.

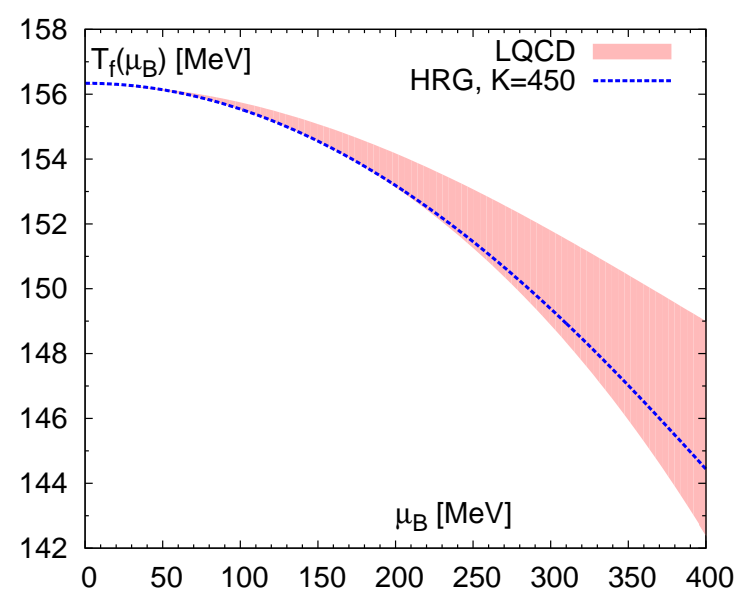

Figure 4: The freeze-out temperature obtained in HRG with repulsive mean-field (blue line) compared to lattice QCD results [8] shown as a band. The width of the band correspond to the uncertainty of the lattice result.

It is reasonable to assume that the freeze-out in heavy ion collisions happens at fixed energy or entropy density as the collision energy is varied. Lower collision energies typically correspond to lower smaller $\mu_{B}$. Therefore, one can define a line of constant energy density that characterizes the freeze-out condition [8]. We assume that freeze-out corresponds to the energy density of $330 \mathrm{MeV} / \mathrm{fm}^{3}$. The freeze-out temperature corresponding to this energy density is shown in Fig. 3 as function of the baryon chemical potential. Here we consider the case of $\mu_{S}=0$ and the case of $n_{S}=0$. The latter being more relevant for the heavy ion collisions. As one can see from the 
Fig. 3 the later corresponds to larger freeze-out temperature. The repulsive mean-field increases the freeze-out temperature a bit. This effect is larger for larger values of $\mu_{B}$ reaching about $3 \mathrm{MeV}$ at the highest $\mu_{B}$. We also calculated the baryon density corresponding to the freeze-out condition. The results are shown in Fig. 3 (right). The repulsive mean field decreases the net baryon density of the freeze-out by about $15 \%$ for $\mu_{S}=0$ and by about $10 \%$ for $n_{S}=0$. Finally we compare the freeze-out temperature calculated in our repulsive mean field model with the results of recent lattice QCD calculations in Fig. 4. The lattice results are shown as band. We see that the freezeout temperature obtained in our model agrees with the lattice given the uncertainties of the latter. Therefore, the repulsive baryon interactions play an important role in the freeze-out in heavy ion collisions.

\section{Conclusion}

In this contribution we considered the role of repulsive interactions in the hadron resonance gas. We compared the virial expansion and the mean-field approach for the treatment of the repulsive interactions and showed that at relatively low densities the two approaches are similar. From this comparison we could determine the size of the mean field. We have used the mean field approach to calculate the equation of state for $T \leq 170 \mathrm{MeV}$ and $\mu_{B} \leq 400 \mathrm{MeV}$. We have found that for the baryon chemical potential $\mu_{B}=300-400 \mathrm{MeV}$ the effect of the effects of the repulsive interactions are quite significant. To consider thermodynamics at larger $\mu_{B}$ it will be necessary to go beyond the expanded version of the mean-field approach considered here. We also plan to extend the mean-field approach for excited baryons. Finally, the effect of hadrons that are predicted by quark models and lattice QCD on the thermodynamic quantities should also be considered.

\section{Acknowledgment}

This work was supported by U.S.Department of Energy under Contract No. DE-SC0012704, and by National Science Center, Poland, under grant Polonez DEC-2015/19/P/ST2/03333 receiving funding from the European Union's Horizon 2020 research and innovation program under the Marie Skłodowska-Curie grant agreement No 665778.

\section{References}

[1] H.-T. Ding, F. Karsch, and S. Mukherjee, Int. J. Mod. Phys. E24, 1530007 (2015), 1504.05274.

[2] P. Petreczky, J. Phys. G39, 093002 (2012), 1203.5320.

[3] R. Venugopalan and M. Prakash, Nucl. Phys. A546, 718 (1992).

[4] M. Albright, J. Kapusta, and C. Young, Phys. Rev. C92, 044904 (2015), 1506.03408.

[5] V. Vovchenko, M. I. Gorenstein, and H. Stoecker, Phys. Rev. Lett. 118, 182301 (2017), 1609.03975.

[6] V. Vovchenko et al., (2017), 1707.09215.

[7] P. Huovinen and P. Petreczky, (2017), 1708.00879.

[8] A. Bazavov et al., Phys. Rev. D95, 054504 (2017), 1701.04325. 